\title{
Reviewers for the Annals of Family Medicine through September 2005
}

Peer reviewers are key to advancing scholarship and contributing to the quality of a research journal. Our sincere thanks to the following volunteers who served as peer reviewers for the Annals from October 2004 through September 2005.

\begin{tabular}{|c|c|c|c|c|}
\hline Adrienne Z. Ables & Robert L. Bratton & Steven A. Dosh & Lillian Gelberg & David F. Howarth \\
\hline Trisha L. Acri & Barbara L. Braun & Susan M. Dovey & Darios Getahun & Mary C. Hroscikoski \\
\hline Patricia Adam & Jane E. Brock & Richard L. Dressler & Valerie J. Gilchrist & John Hsu \\
\hline Alan M. Adelman & Sheana S. Bull & Anne L. Dunlop & James M. Gill & Julian T. Hsu \\
\hline Charles Agyemang & Sandra K. Burge & Jennifer J. Eddy & William R. Gillanders & Elbert S. Huang \\
\hline Erin J. Aiello & Edward J. Callahan & Thomas R. Egnew & Dwenda K. Gjerdingen & William J. Hueston \\
\hline James E. Aikens & James F. Calvert Jr & Andrew C. Eisenberg & James L. Glazer & Mark K. Huntington \\
\hline Virginia A. Aita & Patricia A. Carney & Nancy C. Elder & Marshall Godwin & Carmine $\mathrm{U}$. Iacono \\
\hline Zekeriya Akturk & Anthony P. Catinella & Stephen D. Elgert & Marji Gold & Bette K. Idemoto \\
\hline Suzanne M. Allen & Andrew J. Cave & Kristen K. Elliott & G. Kenneth Goodrick & Nicole Isaacson \\
\hline Bader A. Almustafa & William E. Cayley Jr. & Glyn Elwyn & Felicity A. Goodyear-Smith & Carlos R. Jaen \\
\hline Anne P. Ambarian & Frederick M. Chen & John W. Ely & Andrea E. Gordon & Paul A. James \\
\hline Laura Anderko & Heidi T. Chirayath & John W. Epling & Jonathan P. Graffy & Mollie W. Jenckes \\
\hline James E. Andrews & Leanne M. & Ted D. Epperly & Deborah G. Graham & Eva Kahana \\
\hline David C. Anthony & Chrisman-Khawam & Ronald M. Epstein & Mark D. Grant & Alison K. Karasz \\
\hline Katherine M. Appleton & Charles E. Christianson & Paul A. Estabrooks & William D. Grant & David A. Katerndahl \\
\hline Puneet S. Arora & Dana L. Clark & Lance Evans & Inese G. Grava-Gubins & Nancy L. Keating \\
\hline Bruce Arroll & Elizabeth C. Clark & Lyle J. Fagnan & Lisa E. Graves & Robert D. Keeley \\
\hline Bruce A. Bagley & Richard D. Clover & Brooke E. Farley & Larry A. Green & Anthony R. Kendrick \\
\hline Inis J. Bardella & Stephen W. Cobb & Tillman F. Farley & Lee A. Green & Janko Kersnik \\
\hline Linda L. Barnes & Andrew S. Coco & Thomas H. Feeley & Michael E. Green & Rodger S. Kessler \\
\hline Beth Barnet & Adam L. Cohen & Chris Feifer & K. Allen Greiner & Niharika Khanna \\
\hline Jan M. Barnsley & Deborah J. Cohen & Ruth E. Fenske & Frances Griffiths & George E. Kikano \\
\hline Wendy B. Barr & Kathleen Cole-Kelly & Joshua J. Fenton & Gil C. Grimes & Shersten Killip \\
\hline Bruce P. Barrett & Anthony J. Costa & Jeanne M. Ferrante & Jane M. Gunn & Dana E. King \\
\hline Claudia C. Bartz & Glen R. Couchman & Robert L. Ferrer & Rahul Gupta & Diane K. King \\
\hline Nerissa S. Bauer & Myra A. Crawford & Michael D. Fetters & Michael D. Hagen & Scott E. Kinkade \\
\hline Kay A. Bauman & Jennifer Creer & Chris Feudtner & Jeannie Haggerty & John W. Kirk \\
\hline Dennis J. Baumgardner & Renee M. Crichlow & Kenneth S. Fink & Amy Hagopian & Michael C. Klein \\
\hline Elizabeth A. Bayliss & Jesse C. Crosson & Leonard M. Finn & David L. Hahn & Mark A. Knox \\
\hline John W. Beasley & Michael A. Crouch & Kevin Fiscella & Ricardo G. Hahn & Francis P. Kohrs \\
\hline Lorne A. Becker & Brian K. Crownover & Jennifer R. Fishman & Abigail C. Halperin & Noah P. Kondamudi \\
\hline Justin Beilby & Kathleen A. Culhane-Pera & Susan A. Flocke & Jennifer L. Hamilton & Richelle J. Koopman \\
\hline Barbara R. Bellar & Larry Culpepper & David P. Folsom & Lisa J. Harnack & Neil Korsen \\
\hline Diana M. Bensyl & Sue Curry & Samuel N. Forjuoh & T. Michael Harrington & Richard L. Kravitz \\
\hline Linda T. Bilheimer & Timothy P. Daaleman & Chester H. Fox & Stephen E. Hawes & Alex H. Krist \\
\hline Helen J. Binns & Eric S. Daar & Richard M. Frankel & Meg Hayes & Kimberly T. Krohn \\
\hline Reid B. Blackwelder & Barbara J.Daly & Peter Franks & Viking A. Hedberg & Robin L. Kruse \\
\hline Robert P. Blankfield & Paul L. Dassow & Crystal M. Freeman & Steven W. Heim & David Kuo \\
\hline Carol E. Blenning & Esa Davis & Tom R. Freeman & Virginia A. Hemelt & Anton J. Kuzel \\
\hline Erika B. Bliss & Monique Y. Davis-Smith & Linda French & Scott T. Henderson & Jennifer Elston Lafata \\
\hline Richard D. Blondell & Chris B. Del Mar & Edward M. Friedler & Charles E. Henley & James F. Lamb \\
\hline Thomas Bodenheimer & Kelly J. Devers & George E. Fryer & Eric Henley & Forrest Lang \\
\hline Wendy E. Boone & Joseph A. Diaz & Kelly Fryer-Edwards & Daniel P. Hickey & Emily Jane Zaragoza Lao \\
\hline Jeffrey M. Borkan & Vanessa A. Diaz & Cecelia A. Gaffney & John M. Hickner & Anne C. Larme \\
\hline Francesc Borrell-Carrió & Barbara DiCicco-Bloom & Ronald J. Gagne & Paul C. Hicks & JoAnn E. Leavey \\
\hline Richard J. Botelho & James A. Dickinson & James Galliher & T. Patrick Hill & Sharon D. Lee \\
\hline Carol A. Bova & W. Perry Dickinson & Theodore G. Ganiats & Laura E. Hill-Sakurai & Lawrence M. Leeman \\
\hline Robert C. Bowman & L. Miriam Dickinson & Gregory M. Garrison & James D. Holt & Jennifer P. Leiser \\
\hline Linda Boyd & Allen J. Dietrich & Linda Gask & Jodi Summers Holtrop & James E. Lessenger \\
\hline Raymond G. Boyle & Diane M. Dodendorf & Bradley N. Gaynes & Jennifer L. Hoock & Benjamin H. Levi \\
\hline
\end{tabular}




\begin{tabular}{|c|c|c|c|c|}
\hline Jeffrey P. Levine & Daniel L. Meyer & Paul S. Phillips & Dean A. Seehusen & Kathleen Uhl \\
\hline Barcey T. Levy & Baukje Miedema & Karen M. Piegorsch & Karen R. Sepucha & Mark E. Unverzagt \\
\hline Carmen Lewis & Andrea L. Milam & Donna J. Plonczynski & Eric K. Shaw & Trudy van der Weijden \\
\hline Peter R. Lewis & George W. Miller Jr & J. Michael Pontious & Aziz Sheikh & Daniel J. Van Durme \\
\hline $\mathrm{Li} \mathrm{Li}$ & Kelly R. Miller & Douglas M. Post & Karen J. Sherman & Barbara L. Van Horn \\
\hline Angelo A. Licata & James W. Mold & Michael B. Potter & Cleveland G. Shields & Chris van Weel \\
\hline Robert C. Like & Daniel E. Montano & Donya A. Powers & Laura Sices & Theo Verheij \\
\hline Bruce S. Ling & Lynda G. Montgomery & Richard W. Pretorius & Michael J. Sigmon & Robert M. Via \\
\hline Linda H. Linville & Douglas S. Moodie & David W. Price & Laura A. Siminoff & Daniel C. Vinson \\
\hline David G. Litaker & Sylvia A. Moore & Morgan T. Price & Manoj K. Singh & Robert J. Volk \\
\hline Everett E. Logue & Nancy E. Morden & Jacob Prunuske & Keith S. Sinusas & Michael R. Von Korff \\
\hline Richard A. Londo & Anne L. Mounsey & Sandra Puczynski & D’Andrea F. Skipwith & William C. Wadland \\
\hline Randall L. Longenecker & Marco A. Muñiz & Nadeem Qureshi & Mindy A. Smith & Peggy J. Wagner \\
\hline Daniel R. Longo & David J. Muzina & Howard K. Rabinowitz & Susan J. Smith & Howard Waitzkin \\
\hline Daniel S. Longyhore & Marion R. Nadel & Lee J. Radosh & Ariel K. Smits & Ellen R. Wald \\
\hline Glenn A. Loomis & Rollin W. Nagel & Kalyanakrishnan & Leif I. Solberg & Steven E. Waldren \\
\hline Margaret M. Love & Shyamala Nagendran & Ramakrishnan & Stephen J. Spann & Eric M. Wall \\
\hline May Nawal Lutfiyya & Ira S. Nash & Dino W. Ramzi & John B. Standridge & Lorraine S. Wallace \\
\hline Michael V. Maciosek & Donald E. Nease Jr. & Brian V. Reamy & Barbara Starfield & Richard C. Wasserman \\
\hline Calvin J. Maestro Jr. & Jon O. Neher & Barbara D. Reed & Elizabeth Steiner & Jeanette A. Waxmonsky \\
\hline Julie M. Magri & Richard A. Neill & Andrew M. Reibach & Brian Stello & Sally P. Weaver \\
\hline Manfred Maier & Lynne S. Nemeth & Ann B. Reichsman & G. Gayle Stephens & Pamela J. Werb \\
\hline Deborah S. Main & Robert J. Newman & Shmuel P. Reis & Nancy G. Stevens & James J. Werner \\
\hline Arch G. Mainous III & Warren P. Newton & James P. Richardson & Terrence E. Steyer & David R. West \\
\hline Barbara A. Majeroni & J. Michael Niehoff & Nancy Ridenour & Christopher J. Stille & Simon N. Whitney \\
\hline Rita Mangione-Smith & Mary Patricia Nowalk & Cynthia M. Ripsin & Joseph B. Straton & J. Thad Wilkins \\
\hline Katherine L. Margo & Patrick J. O'Connor & Gloria S. Rizkallah & A. Hal Strelnick & Robert L. Williams \\
\hline William H. Markle & Kelly K. O’Malia & Richard G. Roberts & David M. Studdert & Diane B. Wilson \\
\hline Mark R. Marnocha & Kevin C. Oeffinger & W. David Robinson & Jeff L. Susman & Robert J. Winn \\
\hline Frank Martin & Pamela A. Ohman Strickland & William M. Rodney & Andrew L. Sussman & Samuel Y. Wong \\
\hline Kevin B. Martin & A. John Orzano & Michael A. Rodriguez & Alfred F. Tallia & Steven H. Woolf \\
\hline Lynn M. Martire & Wilson D. Pace & Helen F. Roemhild & Robert S. Tan & Douglas C. Woolley \\
\hline Jennifer A. Mayfield & Michael L. Parchman & Eric Rose & Florence K. Tangka & Barbara P. Yawn \\
\hline John R. McConaghy & Robert W. Parker & Amy K. Rosen & Jonathan L. Temte & Denise M. Young \\
\hline Laurie M. McCormick & George R. Parkerson Jr. & Ellen Rosenberg & Tom D. Thacher & Sara E. Young \\
\hline Reuben R. McDaniel Jr. & Bennett L. Parnes & Lewis G. Sandy & David H. Thom & Pamela M. Zahorik \\
\hline Susan H. McDaniel & Parita Patel & John W. Saultz & Andria M. Thomas & Luis E. Zayas \\
\hline Helen E. Mcllvain & Linda J. Patrick-Miller & Robert M. Saywell Jr. & Paul Thomas & Philip Zazove \\
\hline M. Diane McKee & Dale A. Patterson & Joseph E. Scherger & Jonathan N. Tobin & Richard K. Zimmerman \\
\hline Bonnie McRee & Diane R. Pawlowski & Ronald Schneeweiss & Deborah J. Toobert & Therese M. Zink \\
\hline Jack H. Medalie & Kevin A. Pearce & Sarina B. Schrager & Trissa Torres & Terrell W. Zollinger \\
\hline David R. Mehr & Kevin A. Peterson & Sara Schroter & Peter A. Tran & Adam Zolotor \\
\hline Dan J. Merenstein & Kathryn E. Phillips & John G. Scott & Fred G. Tudiver & John P. Zubialde \\
\hline
\end{tabular}

